\title{
A conduta do tratamento medicamentoso por cuidadores de uma instituição de longa permanência para idosos
}

\author{
Medication therapy management by caregivers \\ from a health of institutionalized elderly individuals
}

Recebido em: 04/02/2016

Aceito em: 28/06/2016
Débora Santos Lula Barros ${ }^{1}$; Débora Oliveira Itacaramby ${ }^{1}$;

Dayde Lane Mendonça-Silva ${ }^{2}$; Silvana Nair Leite ${ }^{2}$

${ }^{1}$ Curso de Farmácia. Universidade Católica de Brasília. Campus I. QS 07, Lote 01, EPCT. Águas Claras, DF, Brasil. CEP 71966-700;

${ }^{2}$ Faculdade de Ciências da Saúde, Universidade de Brasilia. Asa Norte, Brasilia, DF, Brasil. CEP 70910-900;, Brasil.

E-mail:debora.farmacia9@gmail.com

\begin{abstract}
Medicines are an important health technology in geriatric care. In this context, the purpose of this study is to analyze and discuss the medication therapy management from the perspective of elderly caregivers in a long term care facility located in the Federal District, Brazil. According to obtained data by conducting a qualitative study which adopted the interview technique with eight caregivers of the Institution, the complexity of pharmacotherapy, work overload, the non-acceptance of drug use by the aged and the lack of knowledge by caregivers are factors that undermine the rationale and operation of the treatment regimens. Caregivers, along with the health team of the institution, have developed coping strategies not to be forgotten in the administration of medication doses, and there are the provision and regular access to those technologies by users. Although pharmaceutical service topic of discussion in institutions is scarce in the literature, due to the aging population and expansion of the supply of these types of services, it is remarkable the need to maintain this issue on the research agenda and technical-scientific production.
\end{abstract}

Keywords: medication therapy management, caregiver, aged, health of institutionalized elderly individuals, pharmaceutical services

\section{RESUMO}

O medicamento constitui uma importante tecnologia em saúde no cuidado gerontológico. Dentro desse contexto, o objetivo desse estudo é analisar e discutir a conduta do tratamento medicamentoso sob a ótica dos cuidadores de idosos de uma instituição de longa permanência situada no Distrito Federal, Brasil. Segundo os dados obtidos por meio da realização de uma pesquisa qualitativa que adotou a técnica de entrevista com as oito cuidadoras da Instituição, a complexidade da farmacoterapia, a sobrecarga de trabalho, a não aceitação de uso dos medicamentos pelos idosos e a falta de conhecimentos pelos cuidadores são fatores que prejudicam a racionalidade e operacionalidade dos esquemas terapêuticos. Os cuidadores, juntamente com a equipe de saúde da Instituição, desenvolveram estratégias de enfrentamento para não haver esquecimento na administração das doses dos medicamentos, assim como haja o provimento e acesso regular às essas tecnologias pelos utentes. Embora a discussão do tema assistência farmacêutica nessas instituições seja escassa na literatura, em virtude do envelhecimento populacional e ampliação da prestação desses tipos de serviços, é notável a necessidade de manutenção desse tema na agenda de pesquisa e produção técnico-científica.

Palavras-chave: conduta do tratamento medicamentoso, cuidador, idoso, instituição de longa permanência, assistência farmacêutica 


\section{INTRODUÇÃO}

O envelhecimento populacional é um fenômeno atual e emergente que protagoniza amplos debates no cenário da saúde coletiva do nosso país. Embora o arcabouço legal do Brasil estabeleça que os cuidados dos idosos devam ser responsabilidade primária das famílias com o devido apoio do Estado, observa-se a ampliação da prestação de serviços de cuidado geriátrico/gerontológico por meio das Instituições de Longa Permanência para Idosos (ILPI) (1).

$\mathrm{O}$ aumento da longevidade contribuiu para mudança no perfil epidemiológico da população brasileira, acarretando maior prevalência de doenças crônicas não transmissíveis que necessitam de tratamento contínuo. Aliado a esse contexto, a presença concomitante de comorbidades e limitações funcionais também colaboram para que a população idosa consuma mais medicamentos se comparado aos outros grupos etários.

Os idosos residentes nas ILPI apresentam riscos aumentados de sofrer erros de medicação, interações medicamentosas, polifarmácia e reações adversas aos medicamentos, por manifestarem em maior extensão doenças limitantes, pré-disposição à fragilidade e à baixa funcionalidade (2).

Os cuidadores estão entre os profissionais de saúde das ILPI que têm importante papel em auxiliar os idosos nas adaptações físicas e emocionais necessárias para o autocuidado (3). Esse profissional dedica-se a diversas funções e, no meio das atividades prestadas pelos cuidadores das ILPIs, encontra-se a Conduta do Tratamento Medicamentoso (CTM), definida pelo Descritor de Ciências da Saúde (DECS) (4) como:

Assistência na conduta e monitoração na terapia de medicamentos para pacientes que recebem tratamento para câncer ou afecções crônicas, como asma e diabetes, consulta com pacientes e suas famílias sobre o uso adequado de medicação; na condução de programas de bem-estar e prevenção de doenças para melhoria da saúde pública; no controle do uso de medicação em vários ambientes.

Sendo o medicamento uma importante tecnologia em saúde para prover os cuidados aos idosos institucionalizados, o objetivo do presente estudo foi analisar e discutir a conduta do tratamento medicamentoso segundo a perspectiva dos cuidadores de idosos que atuam em uma ILPI no Distrito Federal.

\section{MATERIAIS E MÉTODOS}

Tratou-se de um estudo qualitativo que adotou a técnica da entrevista com roteiro semi-estruturado com os cuidadores de idosos que trabalhavam em uma ILPI no DF.

O local de realização da pesquisa possui natureza filantrópica, sendo mantido principalmente por doações de instituições religiosas e famílias dos idosos institucionalizados. A ILPI atua mais de 40 anos em Brasília, na qual é realizada assistência a 32 idosas residentes. Geralmente as idosas são oriundas de outros estados, possuem baixa renda financeira e estão em condição de abandono familiar.

Para essas idosas são oferecidos serviços multiprofissionais, possibilitando a integração dos conhecimentos das áreas de psicologia, nutrição, enfermagem, fisioterapia, serviço social e medicina geriátrica. Embora possua farmácia na ILPI, não é observada a presença de farmacêutico nesse serviço.

Para a seleção da amostra foi seguido os seguintes critérios de inclusão: ser cuidador da ILPI, atuar nos trabalhos relacionados com a medicação, ter idade mínima de 18 anos e aceitar a participação no estudo por meio da assinatura do Termo de Consentimento Livre e Esclarecido (TCLE).

A ILPI recebe o apoio e trabalho voluntário de algumas pessoas da comunidade que atuam como cuidadores informais. Entretanto, as pesquisadoras não realizaram entrevistas com esses tipos de cuidadores, pois a periodicidade de participação no cuidado e as atividades desempenhadas eram heterogêneas no grupo, sendo este um critério de exclusão no recrutamento dos participantes para o estudo.

Os dados foram coletados entre os meses de abril e junho de 2012. Para a realização dos registros das narrativas dos cuidadores foram utilizadas as gravações dos áudios durante as entrevistas.

Para a publicação das diferentes narrativas dos sujeitos participantes dos atendimentos foi atribuída a identificação numérica (cuidador I, cuidador II e assim sucessivamente), para preservar o sigilo das informações.

O projeto de pesquisa foi submetido ao Comitê de Ética em Pesquisa (CEP) da Universidade Católica de Brasília (UCB), sendo concedida a autorização da realização do estudo sob o parecer de número 266.934. 
As informações registradas durante a coleta de dados foram transcritas e analisadas para a identificação de termos e categorias utilizando como referência a técnica de análise temática da análise de conteúdo, seguindo o instrumento de categorização presente na literatura da área (5). Os discursos dos participantes do estudo revelaram os seguintes temas e atividades dentro do escopo da CTM no âmbito da ILPI: acesso aos medicamentos, complexidade da farmacoterapia, esquecimento da administração de doses, resistência de uso dos medicamentos pelas idosas, sobrecarga de trabalho dos cuidadores e falta de conhecimentos sobre medicamentos.

\section{RESULTADOS E DISCUSSÃO}

Caracterização do perfil dos cuidadores - conhecendo os sujeitos do estudo: foram entrevistados oito cuidadores, refletindo a aceitação de todos participantes que preenchiam os critérios de inclusão. O perfil desses cuidadores constituiu por profissionais do gênero feminino (todos participantes), a maioria com idade entre 30 a 40 anos (seis participantes). Também foi prevalente o estado civil casado (seis participantes), dado semelhantes encontrado em outras pesquisas com cuidadores de idosos institucionalizados $(3,6,7)$.

Na ILPI foi constatada que a maioria das cuidadoras trabalhava menos de quatro anos. Esses dados vão ao encontro a um estudo no qual foi demonstrado que o tempo de trabalho dos cuidadores de idosos formais é inferior a cinco anos, independente da natureza das ILPI serem públicas ou privadas (6), como mostra o relato de uma cuidadora que expressa algumas razões do curto período de tempo de trabalho em ILPI:

\begin{abstract}
"Estou aqui há quatro anos, mas achava que não iria aguentar uma semana. É um trabalho que exige muito, pois aqui temos que ser rápidas por conta da rotina. Além do lado psicológico, aqui somos xingadas e acusadas pelas idosas."
\end{abstract}

Quanto ao grau de instrução das cuidadoras, duas possuíam o ensino fundamental incompleto (sete anos de estudo), cinco apresentavam o ensino médio completo (onze anos de estudo, sendo que dessas três eram técnicas em enfermagem, o que acrescenta mais dois anos de estudo, totalizando treze anos para esses indivíduos em questão) e uma cuidadora apresentava superior incompleto (cursava serviço social e já apresentava quatorze anos de estudo).
Não há uma regulamentação específica quanto à formação do cuidador, tampouco existem requisitos para a contratação e atuação nas ILPI (7). Com base nas informações coletadas, foi possível perceber que essa situação condiz com a realidade do local de estudo, pois a formação do grupo era heterogênea e na ILPI não era exigida uma formação específica para atuação como cuidador. Assim, a capacitação desses profissionais se dá na própria prática do cuidado:

\section{"No início foi dificil, ficava igual uma barata tonta, não sabia o que fazer, fui acostumando, ficava olhando as outras cuidadoras. Elas também ia me dizendo o que fazer."}

(cuidadora, IV)

Diante da demanda emergente de cuidadores para atuar na assistência domiciliar e ILPI, é necessária a ampliação da oferta de cursos de capacitação e fomento voltados para a formação específica de cuidadores de idosos. Não obstante, também é fundamental o desenvolvimento de programas de educação continuada para esses atores no âmbito das ILPI, para oferecer qualificação da assistência à saúde no cuidado gerontológico de maneira contínua e sistemática.

A conduta do tratamento medicamentoso e as suas interfaces com o cuidado do idoso institucionalizado: embora a literatura aponte que os idosos no Brasil apresentam problemas em relação ao acesso aos medicamentos $(8,9)$, esses achados não foram encontrados em todos os discursos dos participantes nesse estudo. Segundo a maioria das cuidadoras, os profissionais do serviço desenvolveram estratégias de enfrentamento para garantir a oferta dos medicamentos para os utentes.

Similarmente, no estudo de Oliveira e Novaes (2012), realizado em cinco ILPI do Distrito Federal, os idosos não encontravam dificuldade de acesso aos medicamentos, pois, além desses serviços serem abastecidos pelo componente básico da assistência farmacêutica, essas Instituições por serem em sua maioria de caráter filantrópico, possuía o abastecimento de medicamentos suprido por doações da comunidade e por recursos obtidos de eventos beneficentes (10).

Conforme os achados do estudo supracitado (10), a Instituição estudada, além de receber medicamentos ofertados pelos serviços de assistência farmacêutica do Sistema Único de Saúde (SUS), também recebia o suprimento dessas tecnologias em saúde por meio de aquisições que foram financiadas com recursos provenientes das doações da comunidade. Ademais, a Instituição 
também recebia medicamentos doados pelas famílias e voluntários.

Geralmente a compra dos medicamentos dos idosos atendidos na Instituição se dava, preferencialmente, nas farmácias privadas, credenciadas no programa Farmácia Popular. Quando questionadas se já deixaram de administrar o medicamento por falta de disponibilidade desses produtos na Instituição, a maioria referiu que não, com exceção de duas cuidadoras:

\section{“Jamais! Não é frequente. Às vezes é questão de um a dois dias sem o medicamento."}

(cuidadora IV)

"Sim, que eu me lembre uma vez. Mas quando falta de uma idosa, pega da outra."

(cuidadora VIII)

A necessidade constante de uso de medicamentos por idosos institucionalizados é um propulsor para que os profissionais da ILPI mobilizem esforços para dar continuidade à farmacoterapia dos utentes. Como nas ILPI a responsabilidade de administração e acesso aos medicamentos é da Instituição e não do idoso, problemas de falta de adesão decorrente do acesso precário aos medicamentos pelos pacientes são minimizados (10). Não obstante, ressalta-se que conhecimentos advindos dos serviços técnico-gerenciais da assistência farmacêutica poderiam favorecer a oferta regular desses produtos nas ILPI e, nesse sentido, é importante a incorporação/ atuação do farmacêutico nesse cenário. Oliveira e Novaes (2013) assinalaram que não foi encontrado farmacêutico nas ILPI estudadas, dado similar encontrado no presente estudo (11).

A Lei 13.021 de 8 de agosto de 2014 (12) estabelece no seu capítulo II, artigo 5: "no âmbito da assistência farmacêutica, as farmácias de qualquer natureza requerem, obrigatoriamente, para seu funcionamento, a responsabilidade e a assistência técnica de farmacêutico habilitado na forma da lei". Isto implica a necessidade de incorporação dos farmacêuticos nos serviços de saúde que disponham de farmácia. Assim, não somente por uma questão de cumprimento legal, faz-se necessário o exercício desse profissional nas farmácias das ILPI para qualificação dos serviços prestados ao paciente em cuidado gerontológico.

A complexidade da farmacoterapia tem sido relacionada a distintas características dos regimes terapêuticos, como o número de medicamentos, a frequência das doses, o tempo de tratamento, as instruções para a administração, entre outros aspectos que podem influenciar nos resultados do tratamento medicamentoso (13).

$\mathrm{Na}$ presente pesquisa, foi identificado que no ambiente da ILPI a complexidade do esquema terapeutico era fator que gerava dificuldades na rotina de cuidado das idosas:

“Em relação à quantidade é complicado, porque tem idosa que toma 10 comprimido em um determinado horário e outra idosa toma 1 comprimido. Ai elas ficam, eu quero meu remédio, tá demorando demais, eu quero ir para minha missa, quero minha comida. Ai você tem que medir glicemia de quem é diabético, aplicar insulina, fazer medicação, ajudar na alimentação, fazer curativo e ai nessa correria você perde muito tempo. Quando são só 2 comprimidos para uma idosa beleza, e quando são 10? Ai eu tenho que sentar e olhar comprimido por comprimido, dosagem por dosagem... e como a gente recebe doação se uma idosa tomar um losartana de 100 $m g$, e eu recebo de $50 \mathrm{mg}$, eu tenho que dar 2 de $50 \mathrm{mg}$, pra formar $100 \mathrm{mg}$, né? Ai tem que ter muita atenção."

(cuidadora I)

A simplificação dos regimes terapêuticos previne a polifarmácia e a ocorrência de Problemas Relacionados aos Medicamentos (PRM), facilitando o acesso aos medicamentos realmente necessários para o atendimento do usuário (13). Também é importante destacar que a simplificação e organização sistemática do esquema posológico facilita a dinâmica do cuidado diário do idoso institucionalizado, além de potencializar a segurança na assistência à saúde.

Um estudo de avaliação do uso de medicamentos pela população idosa apontou que o esquecimento é a um fator limitador importante para adesão ao tratamento medicamentoso (14). A reduzida recordação sobre a utilização dos medicamentos pelos idosos é aspecto comum e pode estar relacionado com perda cognitiva patológica ou até mesmo por declínio funcional decorrente do envelhecimento. Assim, esse quadro endossa a importância da responsabilização da administração dos medicamentos pelos cuidadores e outros profissionais de saúde atuantes nas ILPI (15). Quando foram questionadas se já se esqueceram de administrar os medicamentos para as idosas, todas cuidadoras disseram que não. Esses relatos são contrários àqueles encontrados na literatura 
com cuidadores informais de pacientes não instituicionalizados (16):

"A gente não esquece não. Quando uma tá na dúvida a outra vê, confere as coisas... Lá uma fiscaliza e ajuda a outra."

\section{(cuidadora IV)}

No estudo de Oliveira e Novaes (2013) que descreveu o perfil socioeconômico, epidemiológico e farmacoterapêutico de 154 idosos de cinco ILPI de Brasília, foi encontrado que $92,8 \%$ dos idosos com discernimento tomavam seus medicamentos sozinhos, porém sob supervisão dos cuidadores das Instituições (11). Por outro lado, $56,3 \%$ dos idosos sem discernimento dependiam dos cuidadores para administrá-los, em geral, por via parenteral ou enteral (com emprego de materiais médico-hospitalares). Os demais idosos sem discernimento $(43,7 \%)$ possuíam capacidade de deglutição preservada e tomavam os seus medicamentos também sob supervisão dos cuidadores. A classificação com/sem discernimento foi baseada pela capacidade do idoso de fornecer e receber informações corretas e coerentes e essa estratificação se deu pela própria equipe das Instituições estudadas.

A adesão à medicação pode ser favorecida no caso dos idosos institucionalizados em virtude da administração de medicamentos estar sob responsabilidade dos cuidadores e não do próprio idoso (10). Esse processo atenua fatores como esquecimento e suspensão/alteração do esquema terapêutico pelos utentes.

Diante da negação ou menor frequência de esquecimento dos horários de administração dos medicamentos pelos cuidadores formais da ILPI, verifica-se que a institucionalização de idosos pode favorecer a adesão à medicação observando esse aspecto, já que a responsabilidade passa ser compartilhada pelos cuidadores e outros profissionais de saúde. Também cabe lembrar que a conferência por outros profissionais atuantes no cuidado durante o processo de administração reduzem as possibilidades de esquecimento e erros. Contudo, é importante ressaltar que esse processo de supervisão e trabalho coletivo pode ser desenvolvido na assistência domiciliar, minimizando as possibilidades de esquecimento na administração de doses.

A recusa em tomar os medicamentos por alguns idosos foi outro obstáculo mencionado pelas cuidadoras no momento da administração. A renúncia do consumo dos medicamentos pelos utentes também pode vir permeada de discursos agressivos à pessoa do cuidador e, caso haja insistência do profissional na concretização do ato, o quadro de violência pode se tornar mais grave:

\begin{abstract}
"Quando o idoso não quer tomar o medicamento, não adianta. Você pode amassar, pode colocar embaixo da língua, não adianta. Ela te xinga todinha, xinga até sua alma, se você vacilar ela te agride."
\end{abstract}

(cuidadora II)

Esse comportamento do idoso é um fator que leva ao comprometimento da adesão ao tratamento medicamentoso, pois o consumo do medicamento pode não ser concretizado por falta de cooperação do paciente. Dentre os principais motivos da não aceitação dos medicamentos pelos idosos estão: sensação de desconforto gástrico, dificuldades de deglutição, queixa do gosto ou cheiro ruim e/ou administração incômoda $(10,11,13)$.

A administração de medicamentos é uma das tarefas mais complexas e frágeis do cuidado de um idoso (17), o que requer que os cuidadores demandem esforços múltiplos para garantia do uso correto e seguro da farmacoterapia. As alterações comportamentais, a falta de cooperação e compreensão do utente sobre o procedimento e os sintomas psiquiátricos, são aspectos que amplificam a resistência do idoso (15). Assim, os cuidadores devem estar capacitados para lidarem com essas situações e, estimulados em conjunto com a equipe de saúde, desenvolverem estratégias de enfrentamento que possibilitem a administração dos medicamentos e não torne conflituosa a convivência com o idoso.

O despreparo dos trabalhadores que assistem os idosos institucionalizados constitui importante obstáculo que mitiga a qualidade do cuidado, pois além da formação básica, é essencial a educação continuada para o empoderamento de conhecimentos específicos necessários ao atendimento integral (7). Em um estudo com cuidadores de crianças com hipotireoidismo congênito foi discutido que o conhecimento sobre a farmacoterapia é um item imprescindível para adesão à medicação e uso seguro dos medicamentos (18). Consoante a essa proposta, na presente pesquisa as cuidadoras manifestaram desejo de saber mais sobre os medicamentos consumidos pelos idosos:

"Sou cuidadora particular de uma idosa, eu sei quais ela toma, e para que serve os medicamentos. Aqui, eu sei alguns, todos a gente não sabe. Sabe quais são os males, mais saber exatamente para que serve cada medicamento a gente não sabe. Acredito que nenhuma cuidadora sabe."

(cuidadora $\mathrm{V}$ ) 
"A falta de conhecimento dificulta, gostaria de ajudar. No entanto tenho medo de errar, prefiro me neutralizar."

(cuidadora I)

Um estudo realizado com cuidadoras domiciliares mostrou que as mesmas exibiam necessidades de aquisição de orientações quanto às doenças, à terapia medicamentosa, dietas e exercícios físicos (19). Esse quadro ilustra a importância da atuação da equipe interdisciplinar para as trocas de conhecimentos entre os pares, em prol da emancipação de práticas qualificadas para a assistência à saúde do idoso. Alguns autores relataram que na maioria das vezes as ILPI não apresentam pessoal, tampouco recursos materiais e físicos suficientes para o atendimento aos idosos (20). Em algumas, o trabalho é realizado por cuidadores não qualificados para trabalhar com essa população, dada a oferta crescente de empregos nessa área e a diminuta quantidade de pessoas capacitadas no mercado de trabalho (21).

A sobrecarga de trabalho pode prejudicar a qualidade das tarefas realizadas pelos cuidadores da ILPI. Por consequência, a conduta do tratamento medicamentoso pelos cuidadores pode ser afetada, prejudicando a sua execução que deve ser regida de forma efetiva e segura:

\section{"Eu acho que uma técnica é muito pouco por plantão. São 32 idosas, tem horas que uma passa mal, se for no horário de medicação, eu tenho de parar de dar medicação e acudir a idoso que tá passando mal. Depois eu volto e dou o remédio mesmo fora do horário, mas eu tenho que dar."}

(cuidadora III)

A escassez de recursos humanos no setor saúde é uma queixa recorrente, não somente nos estabelecimentos de natureza privada, mas também, e talvez configure situação ainda mais agravante no SUS. Ter recursos humanos em quantidade e qualidade apropriadas além de melhorar a assistência permite que o ambiente ocupacional possua condições adequadas de trabalho, sem prejudicar a saúde física e mental do cuidador e demais profissionais em exercício na área.

\section{CONCLUSÕES}

Os fatores intervenientes sobre a efetivação da conduta do tratamento medicamentoso suscitados pelos cuidadores estão relacionados com os desafios para assegurar uma administração apropriada, tais como a complexidade da farmacoterapia, baixo conhecimento e sobrecarga de trabalho dos cuidadores e a não aceitação do uso dos medicamentos pelos utentes. Em contraposição, fatores como esquecimento dos horários de administração e acesso aos medicamentos são temas que os cuidadores, em articulação com a equipe de saúde, já estão desenvolvendo uma organização de trabalho que seja resolutiva.

Do ponto de vista normativo, a falta de farmacêutico na farmácia da ILPI retrata a ausência de responsável técnico nesse estabelecimento de saúde, contrariando vários dispositivos legais vigentes no País. Não obstante, vale destacar também que a omissão desse profissional prejudica a oferta de uma assistência à saúde qualificada, já que o mesmo desempenha papel chave na promoção da saúde e do uso racional de medicamentos para os utentes institucionalizados.

A conduta do tratamento medicamentoso ultrapassa as questões relativas às que foram descritas no presente estudo. Tendo em vista que esse fenômeno possui dimensão multifacetada e específica na abrangência do cuidado, cujos contextos são complexos e particulares para cada realidade e perfil de utente assistido, é necessária a realização de novas pesquisas com os cuidadores de outras ILPI, a fim de que esses outros aspectos sejam citados e explorados.

\section{REFERÊNCIAS}

1. Camarano AA, Kanso S. As instituições de longa permanência para idosos no Brasil. Rev Bras Est Pop. 2010; 27(1):233-5. DOI: 10.1590/S010230982010000100014

2. Gautério DP, Santos SSC, Pelzer MT, Barros EJ, Baumgarten L. Caracterização dos idosos usuários de medicação residentes em instituição de longa permanência. Rev Esc Enferm USP. 2012; 46(6):1394-9. DOI: $10.1590 /$ S0080-62342012000600016.
3. Ribeiro MTF, Ferreira RC, Ferreira EF, Magalhães CS, Moreira AN. Perfil dos cuidadores de idosos nas instituições de longa permanência de Belo Horizonte, MG. Ciência \& Saúde Colet. 2008;. 13(4):1285-92. DOI: 10.1590/S1413-81232008000400025.

4. Descritores de Ciências da Saúde. Disponível em http:// decs.bvs.br/cgi-bin/wxis1660.exe/decsserver/. 
5. Oliveira DC. Análise de conteúdo temático-categorial: uma proposta de sistematização. Rev Enferm UERJ. 2008; 16(4):569-76.

6. Ribeiro MTF, Ferreira RC, Magalhães CS, Moreira AN, Ferreira EF. Processo de cuidar nas instituições de longa permanência: visão dos cuidadores formais de idosos. Rev Bras Enferm. 2009; 62(6):870-5. DOI: 10.1590/ S0034-71672009000600011.

7. Sampaio AMO, Rodrigues FN, Pereira VG, Rodrigues SM, Dias CA. Cuidadores de idosos: percepção sobre o envelhecimento e sua influência sobre o ato de cuidar. Est Pesq Psicol. 2011; 11(2):550-613.

8. Aziz MM, Calvo MC, Schneider IJC, Xavier AJ, d'Orsi E. Prevalência e fatores associados ao acesso a medicamentos pela população idosa em uma capital do sul do Brasil: um estudo de base populacional. Cad Saúde Pública. 2011; 27(10):1939-1950. DOI: 10.1590/S0102$-311 X 2011001000007$

9. Paniz VMV, Fassa AG, Facchini LA, Bertoldi AD, Piccini RX, Tomasi E, Thumé E, Silveira DS, Siqueira FV, Rodrigues MA. Acesso a medicamentos de uso contínuo em adultos e idosos nas regiões Sul e Nordeste do Brasil. Cad Saúde Pública. 2008; 24(2):267-280. DOI: 10.1590/S0102-311X2008000200005.

10. Oliveira MP, Novaes MRGG. Uso de medicamento por idosos em instituição de longa permanência. Rev Brasil Enferm. 2012; 5(65):737-744. DOI: 10.1590/S003471672012000500004 .

11. Oliveira MPF, Novaes MRCG. Perfil socioeconômico, epidemiológico e farmacoterapêutico de idosos institucionalizados de Brasília, Brasil. Ciência \& Saúde Colet. 2013; 18(4):1069-1078. DOI: 10.1590/S141381232013000400020 .

12. BRASIL. Lei $n^{\circ} 13.021$, de 8 de agosto de 2014. Dispõe sobre o exercício e a fiscalização das atividades farmacêuticas. Disponível em http://www.planalto.gov.br/ccivil_03/_Ato2011-2014/2014/Lei/L13021.htm
13. Acúrcio FA, Silva AL, Ribeiro AQ, Rocha NP, Silveira MR, Klein $\mathrm{CH}$, Rozenfeld S. Complexidade do regime terapêutico prescrito para idosos. Rev Assoc Med Bras. 2009; 55(4):468-74. DOI: 10.1590/S010442302009000400025.

14. Silva CSO, Pereira MI, Yoshitome AY, Neto-Rodrigues JF, Barbosa DA. Avaliação do uso de medicamentos pela população idosa em Montes Claros, Minas Gerais, Brasil. Esc Anna Nery. 2010; 4(14):811-8. DOI: 10.1590/ S1414-81452010000400022.

15. Barros DSL, Mendonça-Silva DL, Leite SN. Conduta do tratamento medicamentoso por cuidadores de idosos. Interface (Botucatu). 2015; 19(54);527-36. DOI: 10.1590/1807-57622014.0055.

16. Gaioli CCLO, Furegato AR, Santos JFL. Perfil de cuidadores de idosos com doença de alzheimer associado à resiliência. Texto \& Contexto - Enferm. 2012; 21(2):150157. DOI: 10.1590/S0104-07072012000100017.

17. Travis SS, Bethea LS, Winn P. Medication administration hassles reported by family caregivers of dependent elderly persons. J Gerontol A Biol Sci Med Sci. 2000; 55(7):412-417. DOI: 10.1093/gerona/55.7.M412.

18. Oliveira FPS, Ferreira EAP. Adesão ao tratamento do hipotireoidismo congênito segundo relato de cuidadores. Psicol Ref Crít. 2010; 23(1):19-28. DOI: 10.1590/ S0102-79722010000100004.

19. Martins JJ et al. Políticas públicas de atenção à saúde do idoso: reflexão acerca da capacitação dos profissionais da saúde para o cuidado com o idoso. Rev Bras Geriatr Gerontol. 2007; 10(3).

20. Creutzberg M, Gonçalves LHT, Sobottka E. A. The economic survival of long stay institutions for impoverished aged people. Rev Latino-Am Enfermagem. 2007; 15:748-54. DOI: 10.1590/S0104-11692007000700006.

21. Kawasaki KD, Diogo MJD. Assistência domiciliaria ao idoso: perfil do cuidador formal - parte II. Rev Esc Enferm USP. 2001; 35(4):320-327. DOI: 10.1590/S008062342001000300009 . 